\title{
La réforme de la formation des enseignants au Maroc
}

Miloud Lahchimi

\section{OpenEdition}

12 Journals

Édition électronique

URL : https://journals.openedition.org/ries/4402

DOI : $10.4000 /$ ries.4402

ISSN : 2261-4265

Éditeur

France Education international

Édition imprimée

Date de publication : 1 septembre 2015

Pagination : 21-26

ISSN : 1254-4590

\section{Référence électronique}

Miloud Lahchimi, «La réforme de la formation des enseignants au Maroc », Revue internationale d'éducation de Sèvres [En ligne], 69 | septembre 2015, mis en ligne le 01 septembre 2017, consulté le 01 juillet 2021. URL : http://journals.openedition.org/ries/4402 ; DOI : https://doi.org/10.4000/ries. 4402

Ce document a été généré automatiquement le 1 juillet 2021.

(c) Tous droits réservés 


\title{
La réforme de la formation des enseignants au Maroc
}

\author{
Miloud Lahchimi
}

1 La formation des enseignants est un sujet toujours problématique, dans les projets de réforme des systèmes éducatifs. L'importance majeure de cet enjeu se manifeste par une tendance à provoquer des débats à l'échelle internationale, qui portent sur les liens entre la qualification des enseignants et les résultats des systèmes scolaires. L'objectif de cet article est d'analyser la réforme de la formation des enseignants au Maroc, pour la situer par rapport aux transformations que connait ce domaine dans d'autres pays, depuis la dernière décennie $\mathrm{du} \mathrm{XX}^{\mathrm{e}}$ siècle. Quels sont les choix organisationnels et pédagogiques retenus? Quel bilan peut-on établir des nouvelles approches adoptées pour une meilleure qualification des enseignants au Maroc?

\section{La formation des enseignants au Maroc avant la réforme actuelle}

Depuis son indépendance en 1956, le Maroc avait étalé la réforme de la formation des enseignants à travers une série de tentatives d'amélioration qui conservaient deux caractéristiques : la centralisation de la gestion du secteur et une approche humaniste disciplinaire. Trois types d'établissements furent dédiés à la formation, selon les cycles d'enseignement scolaire sous tutelle du ministère de l'éducation nationale (MEN).

3 Les écoles normales supérieures (ENS), d'abord appelées "instituts pédagogiques pour l'enseignement secondaire » selon le décret de 1958. Elles prirent le nom d'ENS en 1963, suite à un arrêté qui fixait les modalités d'accès et l'organisation de la formation. Huit écoles étaient alors chargées de la formation des enseignants du lycée et des agrégés, dont deux dédiées à la formation pour l'enseignement technique et une pour l'éducation physique.

Les conditions d'accès et la durée de formation variaient selon la discipline et le cycle. Une année de formation pour les licenciés dans la majorité des disciplines, quatre ans 
pour l'enseignement technique et l'éducation physique, après un concours destiné aux bacheliers. L'accès fut ouvert dans les années quatre-vingts aux enseignants du collège, pour une durée de deux années de formation. Quant à l'architecture pédagogique, les ENS conservaient une conception disciplinaire de l'élaboration de leur dispositif de formation, malgré des tentatives d'amélioration.

5 Les centres pédagogiques régionaux (CPR) furent créés en octobre 1970 pour assurer la formation des enseignants du collège. Ils étaient au nombre de treize avant la dernière réforme, répartis sur treize des seize académies du royaume. Les conditions d'accès et les modalités de formation évoluèrent selon les besoins du système. Ainsi, l'accès fut d'abord ouvert aux élèves du niveau baccalauréat avant que ne soit exigé le baccalauréat sans concours. Cette situation allait perdurer jusqu'en 1986, date à laquelle furent créés deux cycles de formation. Le premier, appelé «cycle général» était ouvert aux bacheliers après concours, pour une durée de deux années de formation. Le deuxième, appelé « cycle pédagogique », exigeait le Diplôme universitaire d'études générales (DEUG), un concours d'accès et une présélection. La durée de formation était limitée à une année seulement. Cette dernière formule fut généralisée pratiquement pour toutes les matières à l'exception de l'éducation musicale. Sur le plan pédagogique, la formation fut marquée par son aspect académique jusqu'en 1997, date d'adoption de la formation par alternance, grâce à un projet de coopération avec la France.

6 Les centres de formation des instituteurs (CFI), dont le nom fut changé après leur création en 1956. Les 34 CFI étaient dédiés à la formation des enseignants du primaire et furent les premières structures mises en place pour la formation des enseignants au Maroc après l'indépendance. Leur évolution illustre l'histoire de la formation des enseignants au Maroc, avec un profil d'entrée qui passa du certificat de l'enseignement primaire au baccalauréat (1980) puis au DEUG (2007). La durée de formation oscilla entre un et deux ans. Le montage pédagogique du dispositif de formation connut un changement au fil du temps, pour adopter en 2007 une approche de formation par alternance.

7 Ainsi, l'évolution du système de formation des enseignants avant la réforme actuelle suscite deux remarques principales :

- au plan organisationnel: on note la dominance d'une structure éclatée, marquée par l'absence de coordination entre les établissements, ce qui engendra une difficulté d'optimisation des ressources déployées, particulièrement en matière de ressources humaines ;

- au plan pédagogique : chaque établissement avait ses propres modalités de formation, les ENS gardant des dispositions de formation académique, tandis que les CPR, après avoir expérimenté l'approche de formation par compétences, étaient revenus à une organisation dans laquelle chaque centre, voire chaque discipline, optait pour sa propre approche pédagogique. Quant aux CFI, ils avaient tendance à favoriser le principe de professionnalisation.

\section{La nouvelle réforme de la formation des enseignants}

Le Maroc a procédé à partir de l'an 2000 à une refonte de son système éducatif à travers une nouvelle réforme qui visait la mise en œuvre des orientations définies par la Charte nationale de l'éducation et de la formation (CNEF). Cette charte préconisait un ensemble d'orientations concernant les principes fondamentaux du système éducatif marocain. 
Elle arrêtait six espaces de rénovation, parmi lesquels l'organisation pédagogique de l'enseignement formel, qui faisait l'objet d'une nouvelle formulation à travers deux grands cycles scolaires préuniversitaires : le cycle préscolaire et primaire d'une durée de 8 ans ; le cycle secondaire collégial et qualifiant de 6 années de scolarité.

En outre, la Charte réservait un «espace » dédié à «l'amélioration de la qualité de l'éducation et de la formation ». Ce dernier déterminait un ensemble de dispositions, notamment : la révision et l'adaptation des programmes et méthodes d'enseignement ; l'adoption d'une nouvelle génération de manuels scolaires; la diversification de l'utilisation des langues.

En concordance avec ces nouveaux principes et orientations, la CNEF avait réservé une place stratégique à la formation des enseignants. À cet effet, le « levier 13 » de la charte faisait le lien entre "l'engagement des enseignants » et «le renouveau de l'école ", à travers un ensemble d'orientations telles que la qualité de formation des enseignants comme une condition de la réussite scolaire; l'intégration de l'ensemble des établissements de formation, «dans le but de mobiliser l'ensemble des potentialités disponibles "; la nécessité d'intégrer la formation continue dans la gestion de la carrière professionnelle des enseignants.

11 Malgré cette urgence et ces principes, la formation était une composante difficile à maîtriser dans le processus de la réforme lancée depuis 2000, ce qui engendra un retard d'une décennie dans sa mise en œuvre, encore en cours au moment du lancement du Plan d'urgence $(\mathrm{PU})$ de la réforme du système éducatif marocain pour la période 2009-2012. Ce Plan d'urgence distinguait quatre mesures majeures pour améliorer la formation des enseignants :

- une définition des critères et des processus de sélection des enseignants du scolaire ;

- la mise en place de « filières universitaires d'éducation » (FUE);

- la création de centres régionaux des métiers de l'enseignement et de la formation (CRMEF) ;

- la mise en œuvre d'une stratégie de formation continue des enseignants.

Le rapprochement entre les choix adoptés par le Maroc et les tendances internationales ${ }^{1}$, en matière de formation des enseignants, permettent de constater, d'une part, que le Maroc s'est inscrit dans ces tendances internationales par l'adoption du principe de «l'universitarisation» de la formation initiale des enseignants et que, d'autre part, le pays s'est aussi engagé dans l'application du principe de professionnalisation de la formation.

\section{Les nouvelles dispositions de la réforme de la formation des enseignants marocains}

13 Au plan organisationnel, il faut noter l'accroissement de l'implication des universités avec les FUE (18 accréditations pour l'année 2012). De plus, la formation initiale est assurée dans la majorité des cas par les ENS, qui dépendent désormais des universités. La deuxième phase, appelée "formation qualifiante », est assurée par les 15 nouveaux CRMEF, qui ont remplacé les anciens CPR et CFI. Selon le décret de création, un CRMEF est un " établissement de l'enseignement supérieur qui ne dépend pas de l'université ", mais est "sous tutelle de l'autorité gouvernementale chargée de l'enseignement scolaire ». Le même texte aligne la répartition des centres sur la carte géographique des académies et arrête un ensemble de missions confiées aux CRMEF, telles que: la 
qualification des enseignants; la préparation au concours d'agrégation; la formation des directeurs et des cadres d'appui; l'organisation des sessions de formation continue; la recherche scientifique et pédagogique; la production documentaire et l'élaboration des propositions de projets de réformes en matière de curricula et de formation.

En ce qui concerne la formation du corps enseignant, le texte exige l'obtention de la licence universitaire des études générales et des FUE, et la réussite à un concours d'entrée. Ce dernier est organisé, selon les décisions ministérielles, en deux temps : un examen écrit visant à évaluer les connaissances en éducation et les matières d'enseignement ainsi que les capacités en communication; un entretien de $50 \mathrm{mn}$ devant un jury, afin d'évaluer les capacités de base d'enseignement à travers la présentation d'une situation d'enseignement-apprentissage. Les trois décisions ministérielles insistent sur la maîtrise des deux langues, l'arabe et le français, pour tous les cycles et toutes les matières. Chacun de ces deux examens représente $50 \%$ de la note finale au concours. Le décret de création limite la durée de formation à une année dans deux espaces complémentaires, le CRMEF et l'établissement scolaire, à raison de 26 heures par semaine. L'organisation est basée sur les trois cycles scolaires (préscolaire-primaire; collégial; qualifiant). Cette formation est sanctionnée par un " certificat de qualification éducative».

15 Au plan pédagogique, l'architecture du nouveau dispositif de formation est basée sur trois principes fondamentaux: l'approche par compétences avec une architecture modulaire ; l'alternance fondée sur les activités et les situations professionnelles, qui renforcent la professionnalisation à travers $60 \%$ de la masse horaire allouée à la formation; le paradigme réflexif et dynamique de la logique pratique/théorie/ pratique.

16 Ainsi, le Document cadre des orientations pédagogiques fixe quatre compétences à développer: la planification des apprentissages; la gestion des apprentissages; l'évaluation des apprentissages ; la compétence de la recherche et gestion de projet. Le développement de ces compétences est assuré à travers trois types de modules: des "modules principaux", constitués de ressources en sciences de l'éducation et didactique des disciplines; des "modules d'appui », pour renforcer la maîtrise des compétences professionnelles (communication); des «modules optionnels", à raison de deux pour chaque stagiaire.

17 L'alternance, qui permet d'articuler les activités entre le CRMEF et les établissements scolaires, est administrée selon les paramètres suivants :

- les objectifs, qui commencent par la découverte et se terminent par l'évaluation de la progression des compétences, en passant par l'information; lacontractualisation, l'organisation, le suivi et la confrontation pour une maîtrise réflexive ;

- le temps consacré aux grandes phases de chaque opération de formation;

- la fonction de chaque période d'alternance (prospection, contractualisation, implication, suivi et évaluation);

- les acteurs concernés par les activités de formation programmées, y compris les stagiaires ;

- et enfin les modalités d'exécution, qui varient selon les visites de terrain, les ateliers et l'exploitation des dossiers de suivi personnel.

18 Le nouveau dispositif repose sur quatre types d'évaluation finale: une évaluation écrite, qui porte sur les ressources en rapport avec la matière d'enseignement; un entretien oral, qui évalue les ressources professionnelles du métier d'enseignement; 
des situations professionnalisantes, sous forme de micro enseignement ou jeux de rôle ; des situations professionnelles, dans le cadre de la prise de responsabilité effective dans un établissement scolaire.

\section{Quel bilan de la réforme?}

19 Notre bilan, certes provisoire, est basé sur une étude que nous avons encadrée (Marani, 2013). Au plan organisationnel, malgré les efforts enregistrés, la formation des enseignants au Maroc souffre encore d'un ensemble de carences, à savoir :

- l'absence d'instances de coordination entre les établissements chargés de la formation, malgré les orientations préconisées par la CNEF; un dysfonctionnement organisationnel entre les universités chargées de la formation initiale et les CRMEF pour la formation qualifiante ;

- les nouveaux CRMEF n'ont regroupé que les anciens CFI et CPR, au moment où les ENS furent transférées aux universités, ce qui représente une intégration organisationnelle partielle par rapport aux préconisations stipulées par la CNEF ;

- malgré la décision ministérielle du 4 janvier 2013 au sujet des stages pratiques, des problèmes de coordination demeurent encore entre le CRMEF et les établissements scolaires attachés, ce qui affecte le principe d'alternance; ces difficultés altèrent la qualité du principe de professionnalisation dans les CRMEF ;

- une interprétation «conflictuelle » de l'article 2 du décret de création des CRMEF, qui n'a pas tranché, en ce qui concerne la tutelle des CRMEF, entre le pouvoir central (le ministère) et le pouvoir régional (l'académie);

- des difficultés de gestion interne du CRMEF, entre son siège principal et ses annexes, qui entraînent une sous-exploitation des ressources disponibles ;

- l'absence d'une politique de formation continue adaptée aux besoins réels des enseignants, une formation régie par la logique de l'offre et non par celle de la demande, si bien que la réforme n'a pas pu intégrer la formation continue dans la gestion de la carrière professionnelle des enseignants.

Quant au volet pédagogique, des difficultés persistent toujours pour un rapprochement efficace entre les composantes du dispositif de formation. Ainsi, selon Marani (2013), malgré la différence relevée entre les objectifs de la formation initiale et la qualification, $82 \%$ de stagiaires réclament une redondance d'activités entre la formation initiale universitaire dans les FUE et la qualification professionnelle des CRMEF. La tendance au cours magistral semble continuer à prédominer dans la formation modulaire (selon $87 \%$ des enseignants interrogés) et $65 \%$ des enseignants affirment l'absence de concordance entre les modules et les mises en situations professionnelles. D'autre part, $69 \%$ de stagiaires déclarent ne pas investir leur « projet professionnel personnel » dans les situations professionnelles et $72 \%$ de formateurs notent l'absence "d'un minimum de ressources» en termes de connaissances nécessaires chez les enseignants stagiaires pour assurer leur maîtrise de situations professionnelles - ceci met en cause la répartition horaire entre le temps alloué aux modules et celui réservé aux stages, si bien que le contenu des modules fait l'objet de controverses. Le principe réflexif de formation adopté par le nouveau dispositif reste difficile à réaliser, puisque $76 \%$ des stagiaires questionnés manifestent une implication «faible à très faible» dans leur formation. En revanche, les séances de régulation 
restent une occasion fructueuse pour améliorer les compétences des stagiaires (68\% manifestent leur satisfaction).

Cette lecture des grandes lignes de la réforme de la formation des enseignants au Maroc montre un alignement sur les tendances majeures à l'échelle internationale. Ainsi, le pays reprend la professionnalisation et l'universitarisation comme nouvelles approches de la formation. En parallèle, il conserve le principe de la centralisation du dispositif, à travers la dépendance des nouveaux CRMEF au pouvoir éducatif central. Malgré les progrès enregistrés en matière d'offre pédagogique de formation, le Maroc n'a pas accompagné la réforme par des mesures stratégiques. Cette lacune réside dans l'absence d'une politique de gestion prévisionnelle des compétences du corps enseignant, manifestée par la séparation entre la formation initiale, la qualification et la formation continue.

\section{BIBLIOGRAPHIE}

ALAOUI MARANI A. (2013) : Analyse du dispositif de formation des enseignants aux CRMEF : étude de cas, recherche de fin d'études, Centre de formation des inspecteurs de l'enseignement, Rabat, juin, $109 \mathrm{p}$.

Conseil supérieur de l'éducation et de la formation (2000) : Charte nationale de l'éducation et de la formation, CSEF, Rabat.

Ministère de l'éducation nationale, de l'enseignement supérieur et de la recherche scientifique (2009) : Plan d'urgence de la réforme du système éducatif marocain, MENESRS, Rabat.

Ministère de l'éducation nationale (2011) : Document cadre du nouveau dispositif de formation aux CRMEF, MEN, Rabat.

- (2011) : Arrêté ministériel de création des centres régionaux des métiers de l'éducation et de la formation, $\mathrm{n}^{\circ}$ 2.11.672, 23 décembre, Rabat.

- (2012) : Décision du ministre de l'éducation nationale concernant les modalités et les conditions d'accès aux CRMEF, cycle préscolaire et primaire, $\mathrm{n}^{\circ} 12.2199$, cycle collégial ; $n^{\circ} 12.2201$; cycle qualifiant, $n^{\circ} 12.2200,21$ mai 2012, Rabat.

- (2012) : Décision du ministre de l'éducation nationale concernant les sièges et les annexes des CRMEF, $\mathrm{n}^{\circ}$ 12.1866, 15 mars, Rabat.

\section{NOTES}

1. Voir Revue internationale d'éducation de Sèvres, dossier Former des enseignants [en ligne], $\mathrm{n}^{\circ} 5$, décembre 2010, mis en ligne le $1^{\text {er }}$ décembre 2013 [http://ries.revues.org/930]. 
INDEX

Mots-clés : enseignant, formation des enseignants, réforme

Index géographique : Maroc

\section{AUTEUR}

\section{MILOUD LAHCHIMI}

Miloud Lahchimi est lauréat de l'Université libre de Bruxelles en sciences de l'éducation et diplômé de l'Université de Rouen en ingénierie de formation. Il enseigne actuellement au Centre de formation des inspecteurs de l'enseignement à Rabat, après un parcours de formateur d'enseignants depuis 1991. Il a exercé plusieurs responsabilités en matière de formation des cadres au ministère marocain de l'éducation et dirigé un projet d'appui à l'enseignement fondamental dans le cadre d'un partenariat entre le Maroc et l'Agence française de développement (AFD). En parallèle, il a contribué à des études d'évaluation du système éducatif marocain.milohachimi@yahoo.fr 